\title{
Lyapunov Characterization and Analysis of the Operating Modes of the AC-DC Ćuk Converter
}

\author{
Nikola Petrović* ${ }^{*}$ Aleksandra Lekić ${ }^{*}$ and Dušan Stipanović ${ }^{\dagger}$, Member, IEEE
}

\begin{abstract}
In this paper, all operating modes of the AC-DC Ćuk converter have been modeled as linear-time invariant systems where some of the modes were not only modeled but also detected for the first time. Another feature provided in this paper is that the converter can be modeled and analyzed as a switching dynamical system controlled by an external constant duty-ratio switching and the internal switchings shown to be related to the minimum among the time-derivatives of a common quadratic Lyapunov function. Some representative simulations are provided to illustrate and validate the technical results of the paper.
\end{abstract}

Index Terms-AC-DC Converter, Lyapunov Stability Analysis, Switching Dynamical Systems.

\section{INTRODUCTION}

AC-DC converters or rectifiers are power electronics circuits, which have a wide range of applications, such as switching power supplies, uninterruptible power supplies (UPS), battery chargers, notebook adapters etc. Due to the constant advancement of the technology, the market requires AC-DC converters with high power density, high efficiency, and power factor correction (PFC) close to one, in order to meet the harmonic regulations and standards such as IEC 61000-32/IEC 61000-3-4 [1]-[3]. An AC-DC topology, which can be used to satisfy all these requirements, is a bridgeless PFC Ćuk converter.

What we refer to as the AC-DC Ćuk converter [4]-[7] is a single-stage converter which has three reactive elements and three switches. The converter is shown to have $98 \%$ efficiency and that it can be directly connected to the line voltage. By doing so, the first stage full-bridge rectifier may be excluded, which results in reducing the size of the converter. There has been a number of published results regarding construction of bridgeless single-ended primary-inductor converter (SEPIC) PFC [8]-[10] and Buck-Boost PFC convertor [11], which provide complete design procedures of the described converters. All of the mentioned research efforts relate to PFC converters with high power factor and reduced losses by minimizing the number of switches. This is specially visible in the work related to the construction of bridgeless SEPIC converters and its modified forms [8]-[10], where more recent contributions provide converters with a smaller number of switches. There have been a lot of research efforts related to PFC boost converters [12]-[15] and the control methods for the bridgeless boost PFC rectifier [16], [17]. Most of the Boost rectifiers

\footnotetext{
Manuscript received ?? ?, ??; revised ?? ??, ?? Corresponding author: N. Petrović (email: p.z.nikola@etf.bg.ac.rs, phone: +381 11 3218311).

* School of Electrical Engineering, University of Belgrade, Belgrade, Serbia

$\dagger$ Coordinated Science Laboratory, University of Illinois, 1308 West Main Street, Urbana, IL 61801, USA
}

are designed for operating in the continuous conduction mode which is easier to control but produces high switching losses. Some of the Boost PFC rectifiers, however, are designed to provide discontinuous conduction mode solutions [18] in order to reduce the capacitor size and improve power factor (PF).

In this paper, we analyze a bridgeless AC-DC Ćuk converter because of its efficiency. Unlike the standard three phase power factor rectifiers that employ Ćuk power stages [19], this AC-DC Cuk converter has reduced losses on the switches. However, similarly to the standard PFC Cuk rectifier, it has the best power factor when operating in the discontinuous conduction mode [20]. As being an interesting research topic and a circuit with very specific operational features, the ACDC Ćuk converter is used as a test circuit for a construction of the Lyapunov equations in order to obtain suitable output filter for the circuit [21]. Also, PFC Ćuk converter's operation and construction of a PID regulator was provided in [20], [22]. As an addition to these works, one of the contributions of this paper is the detection and the characterization of some new operational modes for the converter. In this paper, all switching sequences, which can occur during converter's operation, are derived and both illustrated and validated via simulation and experimental results. Additional contributions of this paper are the computations of the linear-time invariant models for the operation modes, and a characterization of the internal switchings of the converter based on the minimum of the time-derivatives (among the operating modes) of a common quadratic Lyapunov function of a specific structure that fits all the modes. Finally, our complete Lyapunov characterization of the behaviors of the AC-DC Ćuk converter enables new control approaches to its control by using, for example, hysteresis control that was earlier successfully applied to control of the DC-DC Ćuk converter [23], [24]. This statement is particularly based on the fact that the provided Lyapunov analysis covers all switching behaviors of the mentioned converters.

The paper itself is organized as follows. Section II provides the linear time-invariant dynamic models for the circuit's operating regimes which we refer to as subsystems or operating modes. As mentioned earlier, one of the contributions of this paper is that some particular subsystems were detected, which was not possible by using standard steady-state analysis. In Section III, a common quadratic Lyapunov function with a specific structure that fits all the modes, is derived. Simulation results that illustrate the provided technical derivations are given in Section IV. Experimental verification, which shows consistency with the simulation results, is provided in Section V. Finally, some concluding remarks are formulated in Section VI. 


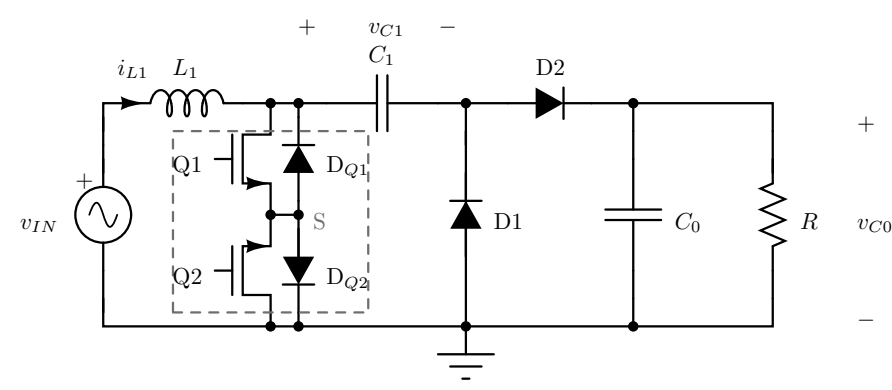

Fig. 1: AC-DC Ćuk converter.

\section{AC-DC ĆUK CONVERTER}

Again, in this paper we consider a particular AC-DC Ćuk converter topology with three reactive elements and three switches, as depicted in Fig. 1. The circuits' state variables are denoted and defined as $\mathbf{x}=\left[\begin{array}{lll}x_{1} & x_{2} & x_{3}\end{array}\right]^{T}=\left[\begin{array}{lll}i_{L 1} & v_{C 1} & v_{C 0}\end{array}\right]^{T}$. The excitation, that is, the circuit's input is the standard AC voltage given as $v_{I N}(t)=V_{m} \sin \left(\omega_{0} t\right)$, where $V_{m}$ is its amplitude and $\omega_{0}$ is its frequency.

While the inductor and capacitors are considered ideal, the conducting transistor and diode are modeled as a series combination of an ideal voltage source and a resistance, annotated as $r_{q}$ and $V_{Q}$ for the transistor and $r_{d}$ and $V_{D}$ for the diode. Furthermore, switch $\mathrm{S}$ is both current and voltage bidirectional and thus realized as a serial connection of two MOSFETs with their body diodes as shown in Fig. 1. When switch $\mathrm{S}$ is turned on, then one MOSFET conducts as well as one body diode and the total voltage drop on the switch is $V_{S}=V_{Q}-V_{D} \approx 0$ and thus ignored. The equivalent resistance of switch $\mathrm{S}$ is $r_{s}=r_{q}+r_{d}$, and in what follows it will be annotated as $r_{s}$.

When the switches are not ideal and switch $\mathrm{S}$ is just about to be turned on, there are three possible outcomes in terms of the circuit's modes depending on the value of the voltage on capacitor $C_{1}$. This happens at moment $t=t_{0}$, when the drive signal to the gates of switch $\mathrm{S}$ becomes logical "1", that is, when the switch turns on. These operating modes or subsystems are collectively denoted by $\widetilde{1}$ and are specified as follows:

1) Subsystem 1: If diodes D1 and D2 are turned off, then only switch $\mathrm{S}$ is conducting and the system matrices are:

$$
\mathbf{A}_{1}=\left[\begin{array}{ccc}
-\frac{r_{s}}{L_{1}} & 0 & 0 \\
0 & 0 & 0 \\
0 & 0 & -\frac{1}{R C_{0}}
\end{array}\right], \quad \mathbf{B}_{1}=\left[\begin{array}{c}
\frac{v_{I N}}{L 1} \\
0 \\
0
\end{array}\right] .
$$

This dynamical system is marginally/boundary stable system with one zero eigenvalue.

Under a practically valid assumption that during one switching period input voltage and output voltage are slowly changing and thus can be considered constant, and assuming that resistance $r_{s}$ has very small value, the solution of differential equations (1) is:

$$
\begin{aligned}
& i_{L 1}(\tau)=\frac{v_{I N}}{L_{1}} \tau+i_{L 1}(0), \\
& v_{C 1}(\tau)=v_{C 1}(0), \\
& v_{C 0}(\tau) \approx\left\langle v_{C 0}\right\rangle_{T_{S}}
\end{aligned}
$$

where $\tau=t-t_{0}$ and $t_{0}$ denotes the beginning of the interval. Initial conditions are denoted as $\mathbf{x}(\tau=0)=\mathbf{x}\left(t=t_{0}\right)$.

2) Subsystem $1 *$ : If at moment $t=t_{0}$, $v_{C 1}\left(t_{0}\right)-r_{S} i_{L 1}\left(t_{0}\right) \geq V_{D 1}$, then diode D1 turns on and the system matrices are given by

$$
\begin{aligned}
\mathbf{A}_{1 *} & =\left[\begin{array}{ccc}
-\frac{r_{s} r_{d 1}}{L_{1}\left(r_{s}+r_{d 1}\right)} & -\frac{r_{s}}{L_{1}\left(r_{s}+r_{d 1}\right)} & 0 \\
\frac{r_{s}}{C_{1}\left(r_{s}+r_{d 1}\right)} & -\frac{1}{C_{1}\left(r_{s}+r_{d 1}\right)} & 0 \\
0 & 0 & -\frac{1}{R C_{0}}
\end{array}\right], \\
\mathbf{B}_{1 *} & =\left[\begin{array}{lll}
\frac{v_{I N}}{L 1}+\frac{r_{s} V_{D 1}}{L_{1}\left(r_{s}+r_{d 1}\right)}, & \frac{V_{D 1}}{C_{1}\left(r_{s}+r_{d 1}\right)}, & 0
\end{array}\right]^{T} .
\end{aligned}
$$

This system is asymptotically stable.

3) Subsystem $1 * *$ : If at moment $t=t_{0}$, $-v_{C 1}\left(t_{0}\right)-v_{C 0}\left(t_{0}\right)+r_{S} i_{L 1}\left(t_{0}\right) \geq V_{D 2}$, then diode $\mathrm{D} 2$ turns on and the system matrices become

$$
\begin{aligned}
\mathbf{A}_{1 * *} & =\left[\begin{array}{lll}
-\frac{r_{s} r_{d 2}}{L_{1}\left(r_{s}+r_{d 2}\right)} & -\frac{r_{s}}{L_{1}\left(r_{s}+r_{d 2}\right)} & -\frac{r_{s}}{L_{1}\left(r_{s}+r_{d 2}\right)} \\
\frac{r_{s}}{C_{1}\left(r_{s}+r_{d 2}\right)} & -\frac{1}{C_{1}\left(r_{s}+r_{d 2}\right)} & -\frac{1}{C_{1}\left(r_{s}+r_{d 2}\right)} \\
\frac{r_{0}\left(r_{s}+r_{d 2}\right)}{C_{0}} & -\frac{1}{C_{0}\left(r_{s}+r_{d 2}\right)} & -\frac{R\left(r_{s}+r_{d 2}\right.}{R\left(r_{s}+r_{d 2}\right) C_{0}}
\end{array}\right], \\
\mathbf{B}_{1 * *} & =\left[\begin{array}{lll}
\frac{v_{I N}}{L 1}-\frac{r_{s} V_{D 2}}{L_{1}\left(r_{s}+r_{d 2}\right)}, & -\frac{V_{D 2}}{C_{1}\left(r_{s}+r_{d 2}\right)}, & -\frac{V_{D 2}}{C_{0}\left(r_{s}+r_{d 2}\right)}
\end{array}\right]^{T} .
\end{aligned}
$$

This system is also asymptotically stable. Furthermore, let us state that to the best of our knowledge, subsystems $1 *$ and $1 * *$ are analyzed for the first time in this paper.

After some time, driver turns off switch $\mathrm{S}$ and in the case when current $i_{L 1}$ is positive, diode D2 turns on. For subsystem $2, \mathrm{~S}$ is turned off, D1 is turned off, D2 is turned on, and the corresponding system matrices are as follows:

$$
\mathbf{A}_{2}=\left[\begin{array}{ccc}
-\frac{r_{d 2}}{L_{1}} & -\frac{1}{L_{1}} & -\frac{1}{L_{1}} \\
\frac{1}{C_{1}} & 0 & 0 \\
\frac{1}{C_{0}} & 0 & -\frac{1}{R C_{0}}
\end{array}\right], \quad \mathbf{B}_{2}=\left[\begin{array}{c}
\frac{v_{I N}-V_{D 2}}{L_{1}} \\
0 \\
0
\end{array}\right] .
$$

This system is asymptotically stable. In general, the converter switches to subsystem 2 if the inductor current is positive or if $i_{L 1}=0$ and $v_{I N}\left(t_{0}\right)-v_{C 1}\left(t_{0}\right)-v_{C 0}\left(t_{0}\right) \geq V_{D 2}$.

Under the assumptions that resistances $r_{s}, r_{d 1}$ and $r_{d 2}$ are negligible and that during one switching period the input voltage can be considered as constant because $T_{S} \ll T_{0}$ and capacitor voltage $v_{C 0} \approx\left\langle v_{C 0}\right\rangle_{T_{S}}$ is very slowly changing, the solution of differential equations (3) is given by

$$
\begin{aligned}
i_{L 1}(\tau)= & A_{1} \sin \left(\omega_{r} \tau\right)+A_{2} \cos \left(\omega_{r} \tau\right), \\
v_{C 1}(\tau)= & v_{I N}-V_{D 2}-\left\langle v_{C 0}\right\rangle_{T_{S}} \\
& -\omega_{r} L_{1}\left(A_{1} \cos \left(\omega_{r} \tau\right)-A_{2} \sin \left(\omega_{r} \tau\right)\right), \\
v_{C 0}(\tau)= & \left\langle v_{C 0}\right\rangle_{T_{S}}
\end{aligned}
$$

where $A_{1}=\frac{v_{I N}-V_{D 2}-\left\langle v_{C 0}\right\rangle_{T_{S}}-v_{C 1}(0)}{\omega_{r} L_{1}}, A_{2}=i_{L 1}(0)$ and $\omega_{r}=\frac{1}{\sqrt{L_{1} C_{1}}}$.

Subsystem 3 occurs when switch $\mathrm{S}$ is turned off and the inductor's current is negative, that is, $i_{L 1}<0$. For this subsystem, $\mathrm{S}$ is off, D1 is on, and D2 is off, resulting in

$$
\mathbf{A}_{3}=\left[\begin{array}{ccc}
-\frac{r_{d 1}}{L_{1}} & -\frac{1}{L_{1}} & 0 \\
\frac{1}{C_{1}} & 0 & 0 \\
0 & 0 & -\frac{1}{R C_{0}}
\end{array}\right], \quad \mathbf{B}_{3}=\left[\begin{array}{c}
\frac{v_{I N}+V_{D 1}}{L_{1}} \\
0 \\
0
\end{array}\right] \text {. }
$$

Now, we can conclude that subsystem 3 is also asymptotically stable. In general, the converter switches to subsystem 3 if the inductor current is negative or if $i_{L 1}=0$ and 
$v_{C 1}\left(t_{0}\right)-v_{I N}\left(t_{0}\right) \geq V_{D 1}$

The solution of differential equations (5) is

$$
\begin{aligned}
i_{L 1}(\tau)= & B_{1} \sin \left(\omega_{r} \tau\right)+B_{2} \cos \left(\omega_{r} \tau\right), \\
v_{C 1}(\tau)= & v_{I N}+V_{D 1} \\
& -\omega_{r} L_{1}\left(B_{1} \cos \left(\omega_{r} \tau\right)-B_{2} \sin \left(\omega_{r} \tau\right)\right), \\
v_{C 0}(\tau)= & \left\langle v_{C 0}\right\rangle_{T_{S}}
\end{aligned}
$$

where $B_{1}=\frac{v_{I N}+V_{D_{1}}-v_{C 1}(0)}{\omega_{r} L_{1}}, B_{2}=i_{L 1}(0)$ and $\omega_{r}=\frac{1}{\sqrt{L_{1} C_{1}}}$.

It is important to note that one additional mode, denoted as subsystem 4, which is a discontinuous current inductor mode, can occur in the following two cases: 1) if the current of the inductor goes to zero after the converter was in subsystem $2 ; 2)$ if the converter was in subsystem 3 and then the inductor current goes to zero. In other words, the converter switches to discontinuous current inductor mode (subsystem 4) when conditions for turning diodes D2 and D3 are no longer satisfied. The system matrices in this subsystem 4 are as follows:

$$
\mathbf{A}_{4}=\left[\begin{array}{ccc}
0 & 0 & 0 \\
0 & 0 & 0 \\
0 & 0 & -\frac{1}{R C_{0}}
\end{array}\right], \quad \mathbf{B}_{4}=\left[\begin{array}{l}
0 \\
0 \\
0
\end{array}\right] .
$$

Subsystem 4 is marginally/boundary stable with two zero eigenvalues. In this subsystem, the inductor current $i_{L 1}(\tau)=i_{L 1}(0)$ and the capacitor voltage $v_{C 1}(\tau)=v_{C 1}(0)$ are constant. Equivalent circuit diagrams for each subsystem of the Ćuk converter are provided in Fig. 2.

\section{A. Switching Sequence}

AC-DC converter which is depicted in Fig. 1 has different switching sequences for positive and negative input voltage. As mentioned in [20], the converter should be designed to operate at the end of the switching period in subsystem 4 , and thus here we provide an analysis of the converter operating in the discontinuous conduction mode. In the case when the input voltage is in its positive half of the line period $T_{0}$, that is, $v_{I N}>0$, at the beginning of the switching period during first subinterval $d T_{S}$, which is generated by the controller, the system operates as one of the $\widetilde{1}$ subsystems. During this interval inductor current increases and becomes positive, while voltage $v_{C 1}$ remains the same. Then, switch $\mathrm{S}$ turns off, and current $i_{L 1}$ is greater than zero, so diode D2 turns on and system continues operating as subsystem 2 . When the inductor current $i_{L 1}$ goes to zero, D2 turns off, but the resonant part of the circuit consisting of inductor $L_{1}$ and capacitor $C_{1}$ forces diode D1 to turn on. Then for some time, until current $i_{L 1} \leq 0$ reaches again zero, the converter operates in subsystem 3 . Then, if the condition for entering subsystem 4 is not satisfied, meaning that the diode D2 turns on, the system can enter subsystems 2 followed by operating in subsystem 3 for a number of times. The converter switches to discontinuous inductor current mode (that is, subsystem 4) when conditions for turning diodes D2 and D3 are no longer satisfied. Thus, for positive input voltage expected switching sequence is $\widetilde{1} \rightarrow 2 \rightarrow\{\{3\} \rightarrow\{2\}\} \rightarrow 4$, where sequence in brackets can either occur multiple times or it doesn't occur at all. For example, during the change of the input voltage from negative to positive values, the expected sequence is $\widetilde{1} \rightarrow 2 \rightarrow 4$.
During the other half of the period when $v_{I N}<0$, the switching sequence is similar as for the positive input voltage. This means that during the first subinterval lasting $d T_{S}$, the converter again operates in subsystem $\widetilde{1}$, but now inductor current $i_{L 1}$ is negative. After that, the converter enters subsystem 3 and then possibly subsystem 2 with the same condition $i_{L 1}=0$. Subsystem 4 again occurs only if the period is long enough so current $i_{L 1}$ reaches zero for the second time. Switching sequence in this case is $\widetilde{1} \rightarrow 3 \rightarrow\{\{2\} \rightarrow\{3\}\} \rightarrow 4$.

In Fig. 3, we provide common switching sequences of the AC-DC Ćuk converter for the two state variables: inductor current $x_{1}$ and capacitor voltage $x_{2}$. The output capacitor voltage is not depicted because its value change, during one switching period, is negligible. In Fig. 3a-c, we plot the variables during one switching period when the input voltage is positive, while in Fig. 3d-f, we provide time diagrams when the input voltage is negative.

\section{B. Operation in the Steady-State}

According to [20] this converter should be designed to work in the discontinuous conduction mode, which means that during one fixed switching period $T_{S}=1 / f_{S}$ it should switch among subsystems $\widetilde{1}, 2,3$ and 4 . The converter, before reaching its steady-state operation, can experience switching sequences which do not include subsystem 4 , but after it reaches the steady-state, it should always switch to subsystem 4 at the end of the period. That means that the sequence $\widetilde{1} \rightarrow 2 \rightarrow 3$ when $v_{I N}>0$ and the sequence $\widetilde{1} \rightarrow 3 \rightarrow 2$ when $v_{I N}<0$ can occur until the converter reaches its steady-state (see Fig. $3 \mathrm{~b}$ and $3 \mathrm{e}$ ).

In the following subsections, the converter's behavior operating in the steady-state will be analyzed in order to explicitly compute the converter's equilibrium. Therefore, only sequences $\widetilde{1} \rightarrow 2 \rightarrow 3 \rightarrow 4$ or $\widetilde{1} \rightarrow 2 \rightarrow 4$ for $v_{I N}>0$ and $\widetilde{1} \rightarrow 3 \rightarrow 2 \rightarrow 4$ or $\widetilde{1} \rightarrow 3 \rightarrow 4$ for $v_{I N}<0$ will be considered. Similar analysis can be derived when there are multiple times the system enters subsystems 2 and 3 (for example $\widetilde{1} \rightarrow 2 \rightarrow 3 \rightarrow 2 \rightarrow 4$ ) and the outcomes will be the same.

1) Positive Input Voltage $v_{I N}>0$ : According to the described switching sequence, when $v_{I N}>0$, then the system during one switching period always switches $\widetilde{1} \rightarrow 2$ and then it can switch to subsystem 3 or 4 . In the subsystem denoted as $\widetilde{1}$, the converter stays after the initial time $t=t_{0}$ for the duration of $\tau_{1}=d T_{S}$. If the capacitor voltage has at the beginning of the switching period initial value $v_{C 1}\left(t_{0}\right) \geq V_{D 1}$, then the converter enters subsystem $1 *$. If the condition $v_{C 1}\left(t_{0}\right) \geq V_{D 1}$ isn't satisfied, then the converter operates in subsystem 1 . In subsystem $1 *$, for a very short interval of time (because of rather small resistances $r_{s}$ and $r_{d 1}$ and thus small time constants), capacitor voltage $v_{C 1}$ becomes $v_{C 1}\left(t_{0}^{+}\right)=V_{D 1}$. The moment when capacitor voltage $v_{C 1}$ equals diode voltage drop is denoted as $t=t_{0}^{+}$because the duration of this time interval is negligible comparing to the duration of the switching period. However, both operations in subsystem 1 and $1 *$ have solutions of the corresponding differential equations 


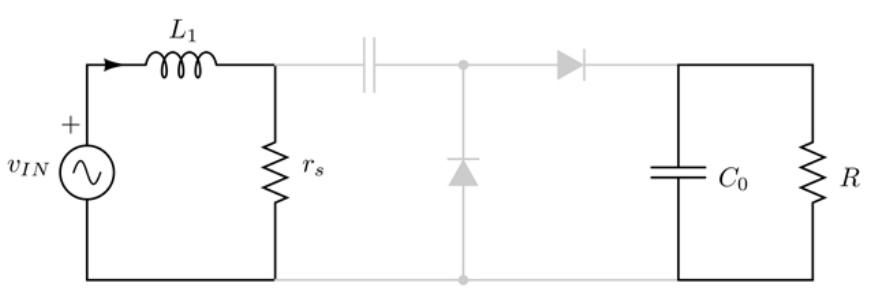

a)

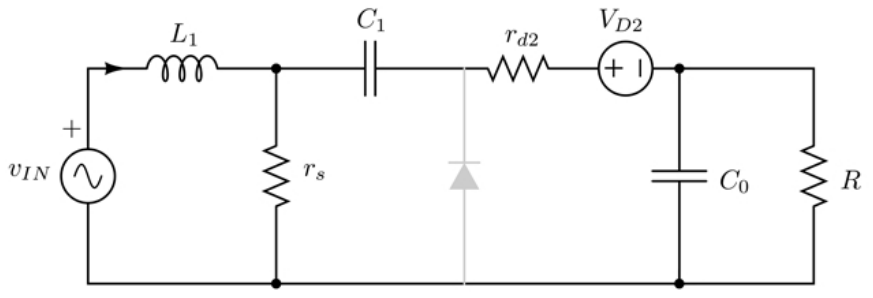

c)

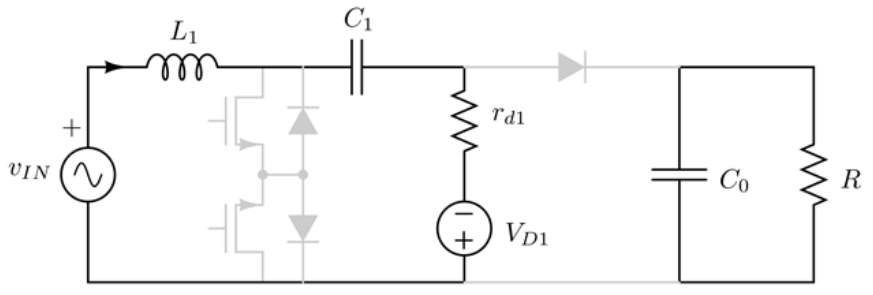

e)

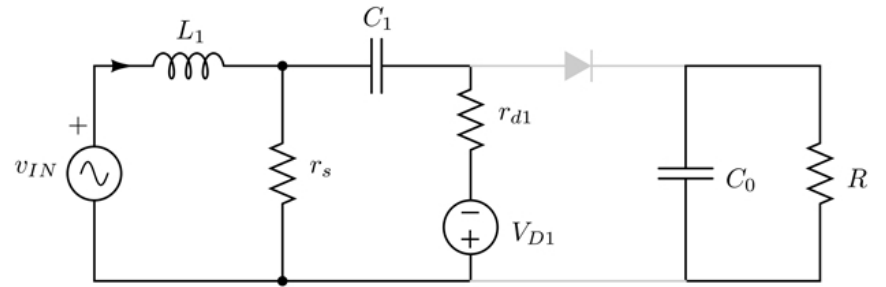

b)

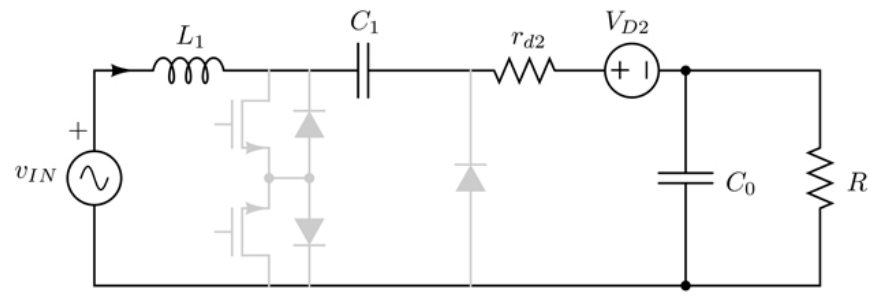

d)

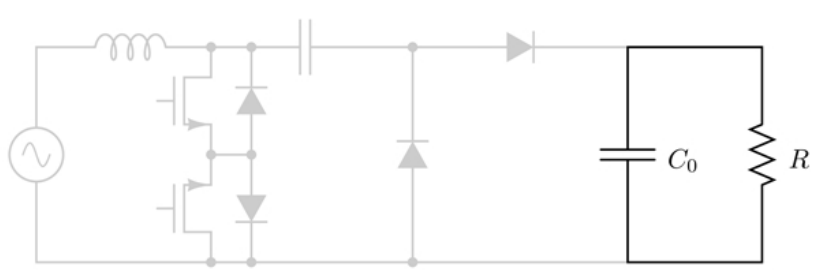

f)

Fig. 2: Equivalent circuit diagrams for the Ćuk converter operating in: a) subsystem 1; b) subsystem $1 *$; c) subsystem $1 * *$; d) subsystem 2; e) subsystem 3 and f) subsystem 4 .

as given in (2) and values at the end of interval $t_{1}=t_{0}+\tau_{1}$ are $v_{C 1}\left(t_{1}\right)=v_{C 1}\left(t_{0}^{+}\right)$(where $v_{C 1}\left(t_{0}^{+}\right)=V_{D 1}$ if the converter operated in subsystem $1 *$ at the beginning of the switching period, or $v_{C 1}\left(t_{0}^{+}\right)=v_{C 1}\left(t_{0}\right)$ if it operated in subsystem 1) and $i_{L 1}\left(t_{1}\right)=\frac{v_{I N} d T_{S}}{L_{1}}+i_{L 1}\left(t_{0}\right)$.

In subsystem 2, the inductor current decreases until it reaches zero. It can be calculated that the duration of this interval of time is $\tau_{2}=\frac{1}{\omega_{r}} \arctan \left(-\frac{A_{2}}{A_{1}}\right)$ and with this value can be determined capacitor $C_{1}$ 's voltage at the end of operation in the subsystem 2 as $v_{C 1}\left(t_{2}\right)=v_{I N}-V_{D 2}-\left\langle v_{C 0}\right\rangle_{T_{S}}+\omega_{r} L_{1} \sqrt{A_{1}^{2}+A_{2}^{2}}$,

$$
\begin{aligned}
& v_{C 1}\left(t_{2}\right)=v_{I N}-V_{D 2}-\left\langle v_{C 0}\right\rangle_{T_{S}}+ \\
& \sqrt{\left(v_{I N}-v_{C 1}\left(t_{1}\right)-V_{D 2}-\left\langle v_{C 0}\right\rangle_{T_{S}}\right)^{2}+\left(\alpha v_{I N}\right)^{2}},
\end{aligned}
$$

where $\alpha=\omega_{r} d T_{S}$.

In the case when after the operation in subsystem 2, the converter enters subsystem 3 , from equations (6) it can be determined that $B_{1}=\frac{v_{I N}+V_{D 1}-v_{C 1}\left(t_{2}\right)}{\omega_{r} L_{1}}$ and $B_{2}=0$. Subsystem 3 operation lasts until the inductor current reaches zero which is satisfied for $\tau_{3}=\frac{\pi}{\omega_{r}}$. At the end of subsystem 3 operation, the capacitor voltage $v_{C 1}\left(t_{3}\right)=2\left(v_{I N}+V_{D 1}\right)-v_{C 1}\left(t_{2}\right)$. If the sum of all intervals satiesfies $\tau_{1}+\tau_{2}+\tau_{3}<T_{S}$, then the converter enters subsystem 4 , in which inductor current $i_{L 1}$ and capacitor voltage $v_{C 1}$ remains constant.

After operating in subsystem 2, the converter enters subsystem 4 if $v_{C 1}\left(t_{2}\right)<v_{I N}+V_{D 1}$, resulting in the following condition:

$$
0<v_{I N}<\frac{2\left(V_{D 1}+V_{D 2}+\left\langle v_{C 0}\right\rangle_{T_{S}}\right)}{\alpha^{2}+1}
$$

for forming switching sequence $\widetilde{1} \rightarrow 2 \rightarrow 4$. This sequence occurs when the input voltage value changes from negative to positive and vice versa.

Now, it is of interest to investigate for which value of the input voltage at the beginning of the period $t=t_{0}$, the converter operates in subsystem $1 *$. In order for the converter to enter subsystem $1 *$ it must be satisfied that the capacitor voltage $v_{C 1}$ is greater than $V_{D 1}$ (inductor current is then zero) at the end of the previous period. This means $v_{C 1}\left(t_{3}\right)>V_{D 1}$, and results in

$$
\begin{aligned}
& v_{C 1}\left(t_{3}\right)=2\left(v_{I N}+V_{D 1}\right)-\left(v_{I N}-V_{D 2}-\right. \\
& \left.5 \sqrt{\left(v_{I N}-V_{D 1}-V_{D 2}-\left\langle v_{C 0}\right\rangle_{T_{S}}\right)^{2}+\left(v_{I N} \omega_{r} d T_{S}\right)^{2}}\right) \\
& >V_{D 1}
\end{aligned}
$$

providing the following limitation:

$$
0<v_{I N}<\frac{4\left(V_{D 1}+V_{D 2}+\left\langle v_{C 0}\right\rangle_{T_{S}}\right)}{\alpha^{2}} .
$$

When $v_{I N}$ doesn't satisfy the previous inequality, then the converter operates with the sequence $1 \rightarrow 2 \rightarrow 3 \rightarrow 4$. Then, the value of voltage $v_{C 1}$ is the same at the beginning of the period as at the end of the period, being $v_{C 1}\left(t_{0}\right)=v_{C 1}\left(t_{3}\right)=v_{C 1}\left(t_{0}+T_{S}\right)$. Using the same analysis 

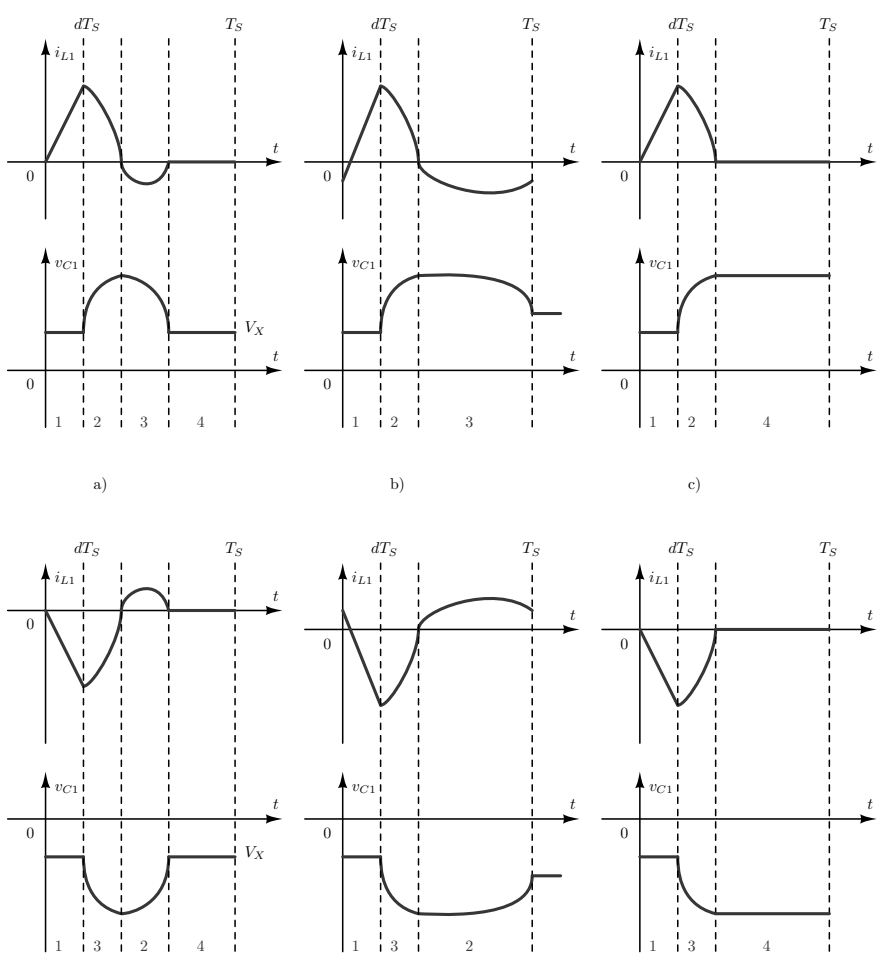

d)

e)

Fig. 3: Time diagrams for the state variables $x_{1}$ and $x_{2}$ for the following switching sequences: a) $1 \rightarrow 2 \rightarrow 3 \rightarrow 4$; b) $1 \rightarrow 2 \rightarrow 3$; c) $1 \rightarrow 2 \rightarrow 4$; d) $1 \rightarrow 3 \rightarrow 2 \rightarrow 4$; e) $1 \rightarrow 3 \rightarrow 2$ and f) $1 \rightarrow 3 \rightarrow 4$.

as before we can compute

$$
\begin{gathered}
v_{C 1}\left(t_{0}\right)=\frac{-v_{I N}^{2} \alpha^{2}+4 v_{I N}\left(\left\langle v_{C 0}\right\rangle_{T_{S}}+V_{D 1}+V_{D 2}\right)}{4\left(\left\langle v_{C 0}\right\rangle_{T_{S}}+V_{D 1}\right)}+ \\
\frac{4\left(V_{D 1}^{2}+V_{D 1} V_{D 2}+\left\langle v_{C 0}\right\rangle_{T_{S}} V_{D 1}\right)}{4\left(\left\langle v_{C 0}\right\rangle_{T_{S}}+V_{D 1}\right)}
\end{gathered}
$$

The analysis done so far is to determine the inductor current waveform. Clearly, the average value of the inductor current during one switching period can be computed as

$$
\begin{aligned}
\left\langle i_{L 1}\right\rangle_{T_{S}}= & \frac{1}{T_{S}}\left(\int_{t_{0}}^{t_{1}} \frac{v_{I N} t}{L_{1}} d t+C_{1} \int_{t_{1}}^{t_{3}} d v_{C 1}\right) \\
& =\frac{v_{I N} d^{2} T_{S}}{2 L_{1}}+\frac{C_{1}}{T_{S}}\left(v_{C 1}\left(t_{3}\right)-v_{C 1}\left(t_{1}\right)\right) .
\end{aligned}
$$

From equation (12) it can be seen that in the case when the converter doesn't enter subsystem $1 *$, the average inductor current has clearly a sine waveform. But in the case when the converter enters subsystem $1 *$, then the average of the inductor current is given by

$$
\begin{aligned}
\left\langle i_{L 1}\right\rangle_{T_{S}} & =\frac{v_{I N} d^{2} T_{S}}{2 L_{1}}+\frac{C_{1}}{T_{S}}\left(v_{I N}+V_{D 1}+V_{D 2}+\left\langle v_{C 0}\right\rangle_{T_{S}}\right. \\
& \sqrt{\left.\left(v_{I N}-V_{D 1}-V_{D 2}-\left\langle v_{C 0}\right\rangle_{T_{S}}\right)^{2}+\left(v_{I N} \alpha\right)^{2}\right)} .
\end{aligned}
$$

2) Negative Input Voltage $v_{I N}<0$ : When the input voltage has a negative value $v_{I N}<0$, then the switching period always starts with switching $\widetilde{1} \rightarrow 3$ and then the converter

switches to subsystem 3 or 4 . When switch $\mathrm{S}$ turns on at the moment $t=t_{0}$, then two different operating modes can occur: 1) mode $1 * *$ if $-v_{C 1}\left(t_{0}\right)-v_{C 0}\left(t_{0}\right) \geq V_{D 2}$ under assumption that $i_{L 1}\left(t_{0}\right)=0$; or 2) mode 1 if this condition is not satisfied. Subsystem $1 * *$ forces capacitor voltage to become $v_{C 1}\left(t_{0}^{+}\right)=-V_{D 2}-\left\langle v_{C 0}\right\rangle_{T_{S}}$. Negligible resistances $r_{s}$ and $r_{d 2}$ give very small time constants, which cause that change capacitor voltage from value $v_{C 1}\left(t_{0}\right)$ to value $v_{C 1}\left(t_{0}^{+}\right)$ occurs almost instantly. Thus, the moment when the capacitor voltage changes is denoted as $t=t_{0}^{+}$. After this change, all state variables trajectories can be computed as in equation (2).

At the end of operation in the subsystem $1 * *$ or subsystem 1, we have $t=t_{1}=t_{0}+d T_{S}$ and $i_{L 1}\left(t_{1}\right)=\frac{v_{I N} d T_{S}}{L_{1}}+i_{L 1}\left(t_{0}\right), v_{C 1}\left(t_{1}\right)=v_{C 1}\left(t_{0}^{+}\right)$and $v_{C 0}\left(t_{1}\right)=\left\langle v_{C 0}\right\rangle_{T_{S}}^{L_{1}}$. The converter then switches to subsystem 3 and its behaviour is given by equation (4) where $B_{1}=\frac{v_{I N}+V_{D 1}-v_{C 1}\left(t_{1}\right)}{\omega_{r} L_{1}}$ and $B_{2}=i_{L 1}\left(t_{1}\right)$. Subsystem 3 ends when the inductor current becomes $i_{L 1}\left(t_{2}\right)=0$ so the duration of this interval is $\tau_{2}=\frac{1}{\omega_{r}}$ atan $\left(-\frac{B_{2}}{B_{1}}\right)$ and the capacitor voltage at the end of the interval is given by

$v_{C 1}\left(t_{2}\right)=v_{I N}+V_{D 1}-\sqrt{\left(v_{I N}+V_{D 1}-v_{C 1}\left(t_{1}\right)\right)^{2}+\left(\alpha v_{I N}\right)^{2}}$.

After operating in subsystem 3, the converter can switch to subsystem 2 with corresponding behavior given in equation (6) with constants $A_{1}=\frac{v_{I N}-V_{D 2}-\left\langle v_{C 0}\right\rangle_{T_{S}}-v_{C 1}\left(t_{2}\right)}{\omega_{r} L_{1}}$ and $A_{2}=0$. This subsystem operation lasts until the inductor current reaches zero for the second time which occurs after $\tau_{3}=\frac{\pi}{\omega_{r}}$. At the end of the converter operating in subsystem 2 we have $i_{L 1}\left(t_{3}\right)=0$ and

$$
v_{C 1}\left(t_{3}\right)=2\left(v_{I N}-V_{D 2}-\left\langle v_{C 0}\right\rangle_{T_{S}}\right)-v_{C 1}\left(t_{2}\right) .
$$

After operating in the subsystem 2, the converter switches to subsystem 4 where according to equation (7) all state variables stay unchanged.

However, the other case is when instead of switching to subsystem 2 and then 4 , the converter switches to subsystem 4 immediately after subsystem 3. This happens when $v_{C 1}\left(t_{2}\right)>-\left\langle v_{C 0}\right\rangle_{T_{S}}-V_{D 2}+v_{I N}$ or

$$
-\frac{2\left(V_{D 1}+V_{D 2}+\left\langle v_{C 0}\right\rangle_{T_{S}}\right)}{\alpha^{2}+1}<v_{I N}<0 .
$$

Sequence $\widetilde{1} \rightarrow 3 \rightarrow 4$ clearly occurs when the input voltage changes from positive to negative values and vice versa.

In the case when at the beginning of the switching period the converter doesn't switch to subsystem $1 * *$, then the capacitor voltage at the end of the switching period has the same value as at the beginning $v_{C 1}\left(t_{0}+T_{S}\right)=v_{C 1}\left(t_{0}\right)$. Using equation (12) it can be concluded that in this case the average value of the inductor current is $\left\langle i_{L 1}\right\rangle_{T_{S}}=\frac{v_{I N} d^{2} T_{S}}{2 L_{1}}$. In the case when converter operates in subsystem $1 * *$, we have the following:

$$
\left\langle i_{L 1}\right\rangle_{T_{S}}=\frac{\frac{v_{I N} d^{2} T_{S}}{2 L_{1}}+\frac{C_{1}}{T_{S}}\left(v_{I N}-V_{D 1}-V_{D 2}-\left\langle v_{C 0}\right\rangle_{T_{S}}\right.}{\left.\sqrt{\left(v_{I N}+V_{D 1}+V_{D 2}+\left\langle v_{C 0}\right\rangle_{T_{S}}\right)^{2}+\left(\alpha v_{I N}\right)^{2}}\right) .}
$$

Again, it can be determined for which values of $v_{I N}$, the converter enters subsystem $1 * *$ by checking condition 
$v_{C 1}\left(t_{3}\right) \leq-V_{D 2}-\left\langle v_{C 0}\right\rangle_{T_{S}}$, which gives the following range for the input voltage:

$$
0>v_{I N} \geq-\frac{4\left(V_{D 1}+V_{D 2}+\left\langle v_{C 0}\right\rangle_{T_{S}}\right)}{\alpha^{2}} .
$$

3) Equilibrium: Equilibrium value for the inductor current can be calculated by averaging $\left\langle i_{L 1}\right\rangle_{T_{S}}$ over the period of the input voltage. From inequalities (10) and (18), it can be seen that the duration of the intervals when converter switches $1 * \rightarrow 2 \rightarrow 3 \rightarrow 4$ or $1 * * \rightarrow 3 \rightarrow 2 \rightarrow 4$, respectively, are the same. Corresponding expressions for the average inductor current are the same in absolute values, but with the opposite sign. Then, $1 \rightarrow 2 \rightarrow 3 \rightarrow 4$ or $1 \rightarrow 3 \rightarrow 2 \rightarrow 4$ last the same and thus $\left\langle i_{L 1}\right\rangle_{T_{S}}=\frac{v_{I N} d^{2} T_{S}}{2 L_{1}}$. Similarly, the computation for the intervals when the switching sequence is $\widetilde{1} \rightarrow 2 \rightarrow 4$ for $v_{I N}>0$ and $\widetilde{1} \rightarrow 3 \rightarrow 4$ for $v_{I N}<0$, results in the same absolute value for $\left\langle i_{L 1}\right\rangle_{T_{S}}$ yet the sign is opposite. By integrating the inductor current $\left\langle i_{L 1}\right\rangle_{T_{S}}$ over period $T_{0}$, intervals when $v_{I N} \geq 0$ and $v_{I N}<0$ are symmetrical yet with opposite signs and thus the overall average value of the inductor current or equilibrium is

$$
\bar{x}_{1}=\bar{i}_{L 1}=0 .
$$

An equilibrium value for the capacitor voltage $v_{C 1}$ over the entire period $T_{0}$ can be computed according to the same approach as for the inductor current. Clearly for both $v_{I N}>0$ and $v_{I N}<0$ all of the time intervals all symmetric. Without complicated calculations and directly from the waveforms it can be concluded that when $v_{I N}>0$, for the most of the time elapsed, we have $v_{C 1}\left(t_{0}\right)=v_{C 1}\left(t_{0}+T_{S}\right)=V_{D 1}$ and for $v_{I N}<0$ we have $v_{C 1}\left(t_{0}\right)=v_{C 1}\left(t_{0}+T_{S}\right)=-V_{D 2}-\left\langle v_{C 0}\right\rangle_{T_{S}}$. For the other times the waveforms integrate symmetrically which produces zero sums. Thus, the equilibrium is $\bar{x}_{2}=$ $\bar{v}_{C 1}=\frac{\frac{T_{0}}{T_{0}}}{T_{0}}\left(V_{D 1}-V_{D 2}-\bar{v}_{C 0}\right)$, or for $V_{D 1}=V_{D 2}$ is

$$
\bar{x}_{2}=-\frac{\bar{x}_{3}}{2} .
$$

To simplify the calculations and final form but without much approximation, we will assume that losses on the conducting switches can be avoided and that the input current waveform can be written as pure sinusoidal $\left\langle i_{L 1}\right\rangle_{T_{S}}=\frac{v_{I N} d^{2} T_{S}}{2 L_{1}}$. Then all of the input power transforms to the output power [20] resulting in $P_{I N}=\frac{V_{m}^{2} d^{2} T_{S}}{4 L_{1}}=\frac{\bar{x}_{3}^{2}}{R}$, and thus

$$
\bar{x}_{3}=M V_{m},
$$

where $M=\sqrt{\frac{R}{2 R_{e}}}$ and $R_{e}=\frac{2 L_{1}}{d^{2} T_{S}}$. Assumptions made for the calculation of the equilibrium are validated in simulations provided in section $\mathrm{V}$.

\section{Lyapunov ANALysis of THE AC-DC ĆUK CONVERTER}

It is well known that for linear switched dynamical systems it is desirable to have a common smooth Lyapunov function. One of the reasons is that one does not need to check the stability on the lines of discontinuity and for more details on this and other benefits we refer to [25] from the purely theoretical point of view and [23], [24] from the application in power electronics point of view. In our case this is feasible and the Lyapunov function form turns out to be diagonal. For the general quadratic Lyapunov function

$$
V(\mathbf{y})=\mathbf{y}^{T} \mathbf{P} \mathbf{y},
$$

we consider the change of variables $\mathbf{y}=\mathbf{x}-\overline{\mathbf{x}}$ where $\overline{\mathbf{x}}$ is an equilibrium. For the quadratic Lyapunov function (22) one can compute its derivative to obtain

$$
\begin{aligned}
\dot{V}_{i}(\mathbf{y}) & =\mathbf{y}^{T}\left(\mathbf{A}_{i}^{T} \mathbf{P}+\mathbf{P} \mathbf{A}_{i}\right) \mathbf{y}+2 \mathbf{y}^{T} \mathbf{P}\left(\mathbf{A}_{i} \overline{\mathbf{x}}+\mathbf{B}_{i}\right)= \\
& =\mathbf{y}^{T} \mathbf{Q}_{i} \mathbf{y}+\mathbf{y}^{T} \mathbf{B}_{y 1},
\end{aligned}
$$

where $\mathbf{Q}_{i}=\mathbf{A}_{i}^{T} \mathbf{P}+\mathbf{P} \mathbf{A}_{i}, \mathbf{B}_{y i}=2 \mathbf{P}\left(\mathbf{A}_{i} \overline{\mathbf{x}}+\mathbf{B}_{i}\right)$ and $i \in$ $\{1,2,3,4\}$.

Assuming that matrix $\mathbf{P}$ is positive definite and of the following form:

$$
\mathbf{P}=\left[\begin{array}{ccc}
p_{1} & p_{12} & p_{13} \\
p_{12} & p_{2} & p_{23} \\
p_{13} & p_{23} & p_{3}
\end{array}\right] \succ 0,
$$

one can derive explicit dependence of entries $p_{1}, p_{12}, p_{13}$, $p_{2}, p_{23}$ and $p_{3}$ on the converter parameters. Considering symmetric negative semidefinite matrix $\mathbf{Q}_{1}$ for subsystem 1:

$$
\mathbf{Q}_{1}=2\left[\begin{array}{ccc}
-\frac{r_{s} p_{1}}{L_{1}} & -\frac{r_{s} p_{12}}{L_{1}} & -\frac{r_{s} p_{13}}{L_{1}} \\
0 & 0 & 0 \\
-\frac{p_{13}}{R C_{0}} & -\frac{p_{23}}{R C_{0}} & -\frac{p_{3}}{R C_{0}}
\end{array}\right] \preceq 0,
$$

in order to preserve symmetry, it should be $p_{12}=p_{23}=0$ and $\frac{r_{s} p_{13}}{L_{1}}=\frac{p_{13}}{R C_{0}}$ which in the general case provides $p_{13}=0$. This results in matrix $\mathbf{P}$ being in the diagonal form.

For subsystem 2, the Lyapunov function derivative is related to the corresponding matrix of the form

$$
\mathbf{Q}_{2}=\left[\begin{array}{ccc}
-\frac{2 r_{d 2} p_{1}}{L_{1}} & \frac{p_{2}}{C_{1}}-\frac{p_{1}}{L_{1}} & \frac{p_{3}}{C_{0}}-\frac{p_{1}}{L_{1}} \\
\frac{p_{2}}{C_{1}}-\frac{p_{1}}{L_{1}} & 0 & 0 \\
\frac{p_{3}}{C_{1}}-\frac{p_{1}}{L_{1}} & 0 & -\frac{2 p_{3}}{R C_{0}}
\end{array}\right],
$$

which is negative semidefinite when $\frac{p_{1}}{L_{1}}=\frac{p_{2}}{C_{1}}=\frac{p_{3}}{C_{0}}$ and with eigenvalues with both polarities when $\frac{p_{1}}{L_{1}}=\frac{p_{2}}{C_{1}}$ and $\frac{p_{1}}{L_{1}} \neq \frac{p_{3}}{C_{0}}$.

For subsystem 3, we obtain

$$
\mathbf{Q}_{3}=\left[\begin{array}{ccc}
-\frac{2 r_{d 1} p_{1}}{L_{1}} & \frac{p_{2}}{C_{1}}-\frac{p_{1}}{L_{1}} & 0 \\
\frac{p_{2}}{C_{1}}-\frac{p_{1}}{L_{1}} & 0 & 0 \\
0 & 0 & -\frac{2 p_{3}}{R C_{0}}
\end{array}\right] \preceq 0
$$

which is negative semidefinite when $\frac{p_{1}}{L_{1}}=\frac{p_{2}}{C_{1}}$. If this result is applied in the equation (26) then negative definite matrix $\mathbf{Q}_{2}$ is obtained for $\frac{p_{1}}{L_{1}}=\frac{p_{3}}{C_{0}}$.

Subsystems 4 has the corresponding matrix in the following form:

$$
\mathbf{Q}_{4}=\left[\begin{array}{cc}
\mathbf{0}_{2 \times 2} & \mathbf{0}_{2 \times 1} \\
\mathbf{0}_{1 \times 2} & -\frac{2 p_{3}}{R C_{0}}
\end{array}\right],
$$

which is clearly negative semidefinite.

From equations (25)-(28), it can be concluded that a common Lyapunov function exists, that is, a common $\mathbf{P}$ exists and is of the form

$$
\mathbf{P}=p\left[\begin{array}{ccc}
L_{1} & 0 & 0 \\
0 & C_{1} & 0 \\
0 & 0 & C_{0}
\end{array}\right], \quad p>0 .
$$


Now, we will proceed by investigating if the obtained form of a common Lyapunov function (29) works for the converter with losses. In particular, we obtain

1) Subsystem 1:

$$
\begin{gathered}
\mathbf{Q}_{1}=2 p\left[\begin{array}{ccc}
-r_{s} & 0 & 0 \\
0 & 0 & 0 \\
0 & 0 & -\frac{1}{R}
\end{array}\right] \preceq 0, \\
\mathbf{B}_{y 1}=2 p\left[v_{I N}, \quad 0, \quad-\frac{\bar{x}_{3}}{R}\right] .
\end{gathered}
$$

2) Subsystem $1 *$ :

$$
\begin{gathered}
\mathbf{Q}_{1 *}=2 p\left[\begin{array}{ccc}
-\frac{r_{s} r_{d 1}}{r_{s}+r_{d 1}} & 0 & 0 \\
0 & -\frac{1}{r_{s}+r_{d 1}} & 0 \\
0 & 0 & -\frac{1}{R}
\end{array}\right] \prec 0, \\
\mathbf{B}_{y 1 *}=2 p\left[\begin{array}{c}
v_{I N}+\frac{r_{s} V_{D 1}}{r_{s}+r_{d 1}}-\frac{r_{s}\left(r_{d 1} \bar{x}_{1}+\bar{x}_{2}\right)}{r_{s}+r_{d 1}} \\
\frac{V_{D 1}-r_{s} \bar{x}_{1}-\bar{x}_{2}}{r_{s}+\frac{r_{d 1}}{\bar{x}_{3}}} \\
\\
-\frac{\bar{x}_{R}}{R}
\end{array}\right] .
\end{gathered}
$$

3) Subsystem $1 * *$ :

$$
\begin{gathered}
\mathbf{Q}_{1 * *}=p\left[\begin{array}{ccc}
-\frac{2 r_{s} r_{d 2}}{r_{s}+r_{d 2}} & 0 & 0 \\
0 & -\frac{2}{r_{s}+r_{d 2}} & -\frac{1}{r_{s}+r_{d 2}} \\
0 & -\frac{1}{r_{s}+r_{d 2}} & -2 \frac{R+r_{s}+r_{d 2}}{R\left(r_{s}+r_{d 2}\right)}
\end{array}\right] \prec 0, \\
\mathbf{B}_{y 1 * *}=2 p\left[\begin{array}{c}
v_{I N}-r_{s} \frac{V_{D 2}+r_{d 2} \bar{x}_{1}+\bar{x}_{2}+\bar{x}_{3}}{r_{s}+r_{d 2}} \\
\frac{-V_{D 2}+r_{s} \bar{x}_{1}-\bar{x}_{2}-\bar{x}_{3}}{r_{s}+r_{d 2}} \\
\frac{R\left(-V_{D 2}+r_{s} \bar{x}_{1}-\bar{x}_{2}\right)-\left(R+r_{s}+r_{d 2}\right) \bar{x}_{3}}{R\left(r_{s}+r_{d 2}\right)}
\end{array}\right] .
\end{gathered}
$$

Subsystem 2:

$$
\begin{gathered}
\mathbf{Q}_{2}=2 p\left[\begin{array}{ccc}
-r_{d 2} & 0 & 0 \\
0 & 0 & 0 \\
0 & 0 & -\frac{1}{R}
\end{array}\right] \preceq 0, \\
\mathbf{B}_{y 2}=2 p\left[v_{I N}-V_{D 2}-\bar{x}_{2}-\bar{x}_{3}, \quad 0 \quad-\frac{\bar{x}_{3}}{R}\right]^{T} .
\end{gathered}
$$

Subsystem 3:

$$
\begin{gathered}
\mathbf{Q}_{3}=2 p\left[\begin{array}{ccc}
-r_{d 1} & 0 & 0 \\
0 & 0 & 0 \\
0 & 0 & -\frac{1}{R}
\end{array}\right] \preceq 0, \\
\mathbf{B}_{y 3}=2 p\left[v_{I N}+V_{D 1}-\bar{x}_{2}, \quad 0, \quad-\frac{\bar{x}_{3}}{R}\right]^{T} .
\end{gathered}
$$

Subsystem 4:

$$
\begin{gathered}
\mathbf{Q}_{4}=2 p\left[\begin{array}{ccc}
0 & 0 & 0 \\
0 & 0 & 0 \\
0 & 0 & -\frac{1}{R}
\end{array}\right] \preceq 0 \\
\mathbf{B}_{y 4}=2 p\left[\begin{array}{llc}
0 & 0 & -\frac{\bar{x}_{3}}{R}
\end{array}\right]^{T}
\end{gathered}
$$

Using the calculations for the equilibrium from the previous section $\overline{\mathbf{x}}=\left[\begin{array}{lll}0 & -\frac{\bar{x}_{3}}{2} \bar{x}_{3}\end{array}\right]^{T}$, where $\bar{x}_{3}=M V_{m}$, the time derivatives of the Lyapunov function are as follows:

$$
\begin{array}{ll}
\dot{V}_{1}(\mathbf{y})= & 2 p\left(-r_{s} y_{1}^{2}-\frac{y_{3}^{2}}{R}+\left(v_{I N}-r_{s} \bar{x}_{1}\right) y_{1}-\frac{\bar{x}_{3} y_{3}}{R}\right), \\
\dot{V}_{1 *}(\mathbf{y})= & 2 p\left(-\frac{r_{s} r_{d 1}}{r_{s}+r_{d 1}} y_{1}^{2}-\frac{y_{2}^{2}}{r_{s}+r_{d 1}}-\frac{y_{3}^{2}}{R}+\right. \\
& \left.\left(v_{I N}+\frac{r_{s}\left(V_{D 1}-\bar{x}_{2}\right)}{r_{s}+r_{d 1}}\right) y_{1}+\frac{V_{D 1}-\bar{x}_{2}}{r_{s}+r_{d 1}} y_{2}-\frac{\bar{x}_{3} y_{3}}{R}\right), \\
\dot{V}_{1 * *}(\mathbf{y})= & 2 p\left(-\frac{r_{s} r_{d 2}}{r_{s}+r_{d 2}} y_{1}^{2}-\frac{y_{2}^{2}}{r_{s}+r_{d 2}}-\frac{y_{3}^{2}\left(R+r_{s}+r_{d 2}\right)}{R\left(r_{s}+r_{d 2}\right)}+\right. \\
& \left.v_{I N} y_{1}-\frac{\left(V_{D 2}+\bar{x}_{2}+\bar{x}_{3}\right)\left(-r_{s} y_{1}+y_{2}+y_{3}\right)}{r_{s}+r_{d 2}}-\frac{\bar{x}_{3} y_{3}}{R}\right), \\
\dot{V}_{2}(\mathbf{y})=\quad 2 p\left(-r_{d 2} y_{1}^{2}-\frac{y_{3}^{2}}{R}+\right. & \left.\left(v_{I N}-V_{D 2}-\bar{x}_{2}-\bar{x}_{3}\right) y_{1}-\frac{\bar{x}_{3} y_{3}}{R}\right), \\
\dot{V}_{3}(\mathbf{y})=\quad 2 p\left(-r_{d 1} y_{1}^{2}-\frac{y_{3}^{2}}{R}\right. & \left.+\left(v_{I N}+V_{D 1}-\bar{x}_{2}\right) y_{1}-\frac{\bar{x}_{3} y_{3}}{R}\right), \\
\dot{V}_{4}(\mathbf{y})=\quad 2 p\left(-\frac{y_{3}^{2}}{R}-\frac{\bar{x}_{3} y_{3}}{R}\right) .
\end{array}
$$

\section{A. Lyapunov function characterization of the state dependent switching}

The first issue to be investigated is to determine or characterize which subsystem occurs when the switch $\mathrm{S}$ is turned on. The analysis which corresponds to this case is the one related to subsystems $1,1 *$ and $1 * *$. Comparing derivatives corresponding to subsystems 1 and $1 *$ results in $\dot{V}_{1}(\mathbf{y})<$ $\dot{V}_{1 *}(\mathbf{y})$ if $x_{2}>\bar{x}_{2}-r_{s} x_{1}$ or $x_{2}<V_{D 1}+r_{s} x_{1}$. This is equivalent to diode D1 turning on. Thus, the converter operates in subsystem 1 if diode D1 cannot conduct. Similarly, for subsystems 1 and $1 * *$ we obtain $\dot{V}_{1}(\mathbf{y})<\dot{V}_{1 * *}(\mathbf{y})$ if $x_{2}+x_{3}>\bar{x}_{2}+\bar{x}_{3}+V_{D 2}+r_{s} x_{1}$ or $x_{2}+x_{3}<V_{D 2}+r_{s} x_{1}$. This provides a condition for diode D2 to turn on. Additionally, subsystem $1 *$ can occur when $v_{I N}>0$, because in that case $x_{2}>0$ and also it can happen that $x_{2} \geq V_{D 1}+r_{s} x_{1} \geq V_{D 1}$. Subsystem $1 * *$ occurs when $v_{I N}<0$ while in this case $x_{2}<0$ and possibly $x_{2}<-x_{3}+V_{D 2}+r_{s} x_{1}$. Therefore, this Lyapunov characterization provides conditions for the system operations in one of the subsystems $1,1 *$ or $1 * *$. As expected and shown, the obtained conditions also satisfy conditions for the corresponding diodes D1 or D2, to conduct.

In order to characterize another state dependent switching which happens after switch $\mathrm{S}$ is turned off, let us first recall that we group three subsystems $1,1 *$ and $1 * *$ as one combined subsystem $\widetilde{1}$. Now, we will show that the state dependent switching after the switch is turned off can be characterized by computing the minimum among the time derivatives of the quadratic Lyapunov function derived in (30) with respect to subsystems 2, 3 and 4.

Theorem 1: After switch $\mathrm{S}$ is turned off, the system will be switching among the subsystems 2,3 and 4 by choosing the subsystem which minimizes $\min _{i \in\{2,3,4\}}\left\{\dot{V}_{i}(\mathbf{y})\right\}$.

Proof: The first switching sequence to analyze is when the input voltage $v_{I N}(t)=V_{m} \sin \left(\omega_{0} t\right)$ is positive. Then, during the first subinterval $n T_{S} \leq t \leq n T_{S}+d T_{S}$, the converter operates in subsystem $\widetilde{1}$. In subsystem $\widetilde{1}$, the inductor current increases, which means that $y_{1}=x_{1}-\bar{x}_{1}=x_{1}>0$. After subsystem $\widetilde{1}$ the converter always enters subsystem 2 . This is because the condition for entering subsystem 2 is $\dot{V}_{2}(\mathbf{y})<$ 
$\dot{V}_{3}(\mathbf{y})$ and $\dot{V}_{2}(\mathbf{y})<\dot{V}_{4}(\mathbf{y})$. First condition $\dot{V}_{2}(\mathbf{y})<\dot{V}_{3}(\mathbf{y})$ implies

$$
\left(r_{d 1}-r_{d 2}\right) y_{1}^{2}+\underbrace{\left(-V_{D 1}-V_{D 2}-\bar{x}_{3}\right)}_{<0} y_{1}<0,
$$

which is under the assumption that D1 and D2 have the same characteristics (that is, parameters) and $y_{1} \geq 0$, which holds after the converter operates in subsystem $\widetilde{1}$. Even if $r_{d 1} \neq r_{d 2}$ for $r_{d 1}>r_{d 2}$, the inequality is satisfied for $0<y_{1}<\frac{V_{D 1}+V_{D 2}+\bar{x}_{3}}{r_{d 1}-r_{d 2}}$, where the upper bound presents a very high inductor current value which is not reachable. On the other hand, if $r_{d 1}<r_{d 2}$, then the inequality $\dot{V}_{2}(\mathbf{y})<\dot{V}_{3}(\mathbf{y})$ is satisfied for $y_{1}<-\frac{V_{D 1}+V_{D 2}+\bar{x}_{3}}{\left|r_{d 1}-r_{d 2}\right|}$ and $y_{1}>0$. After the converter operates in subsystem $\widetilde{1}$ is $y_{1}>0$, the inequality $\dot{V}_{2}(\mathbf{y})<\dot{V}_{3}(\mathbf{y})$ always holds. Inequality $\dot{V}_{2}(\mathbf{y})<\dot{V}_{4}(\mathbf{y})$ is satisfied for

$$
-r_{d 2} y_{1}^{2}+\underbrace{\left(v_{I N}-V_{D 2}-\bar{x}_{2}-\bar{x}_{3}\right)}_{<0} y_{1}<0
$$

providing that $y_{1}>0$ or $y_{1}<\frac{v_{I N}-V_{D 2}-\bar{x}_{2}-\bar{x}_{3}}{r_{d 2}}$. Clearly, when $y_{1}>0$ then $\dot{V}_{2}(\mathbf{y})<\dot{V}_{3}(\mathbf{y})$ plus $\dot{V}_{2}(\mathbf{y})<\dot{V}_{4}(\mathbf{y})$ and after subsystem $\widetilde{1}$, the converter enters subsystem 2 .

Subsystem 2 operation ends when $x_{1}=y_{1}=0$ and then the inductor current decreases. Then, if $y_{1}=0$, all derivatives have the same value $\dot{V}_{2}(\mathbf{y})=\dot{V}_{3}(\mathbf{y})=\dot{V}_{4}(\mathbf{y})$. The condition for entering subsystem 3 or 4 is determined by the condition for diode D1 conducting. If the inductor current is zero, then diode D1 conducts if and only if $x_{2}-v_{I N} \geq V_{D 1}$, or $y_{2} \geq-\bar{x}_{2}-V_{D 1}+v_{I N}$. So, after operating in subsystem 2 , the converter enters subsystem 3 if $y_{2} \geq-\bar{x}_{2}-V_{D 1}+v_{I N}$ and subsystem 4 if that inequality doesn't hold. If however converter enters subsystem 3, it can afterwards operate in subsystem 4 when again $y_{2}<-\bar{x}_{2}-V_{D 1}+v_{I N}$. The converter remains in subsystem 3 while $\dot{V}_{3}(\mathbf{y})<\dot{V}_{4}(\mathbf{y})$ or

$$
y_{1}(-r_{d 1} y_{1}+\underbrace{\left(v_{I N}+V_{D 1}-\bar{x}_{2}\right)}_{>0 \text { for } v_{I N}>0})<0,
$$

which holds for $y_{1}<0$. So after operating in subsystem 3 , the converter enters subsystem 4 when the inductor current reaches zero.

In the case when the input voltage is negative, possible switching sequences are $\widetilde{1} \rightarrow 3 \rightarrow 4, \widetilde{1} \rightarrow 3 \rightarrow 2 \rightarrow 4$ and $\widetilde{1} \rightarrow 3 \rightarrow 2$. Again, externally switch $\mathrm{S}$ is turned on at the beginning of the period and then the converter operates in subsystem $\widetilde{1}$. After operating in subsystem $\widetilde{1}$, the current is negative, $y_{1}<0$, so by inequalities (31) and (33), the converter always enters subsystem 3 .

If the condition $v_{I N}-v_{C 1}-v_{C 0} \geq V_{D 2}$ is satisfied at the end of operation in subsystem 3 when $y_{1}=0$, then the converter enters subsystem 2. If however $v_{I N}-v_{C 1}-v_{C 0}<V_{D 2}$, then after operating in subsystem 3 , the converter operates in subsystem 4 until the end of the period. In the cases when during operation in subsystem 2 it happens that $v_{I N}-v_{C 1}-$ $v_{C 0}=V_{D 2}$, then the converter again enters subsystem 4 .

It should be noted that during the operation in subsystem 4 , the converter cannot enter any other subsystem because all

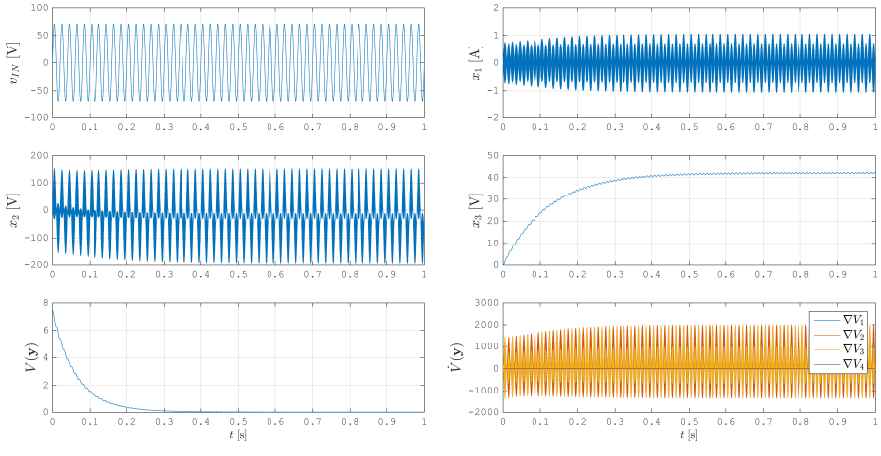

Fig. 4: PFC rectifier's time diagrams.
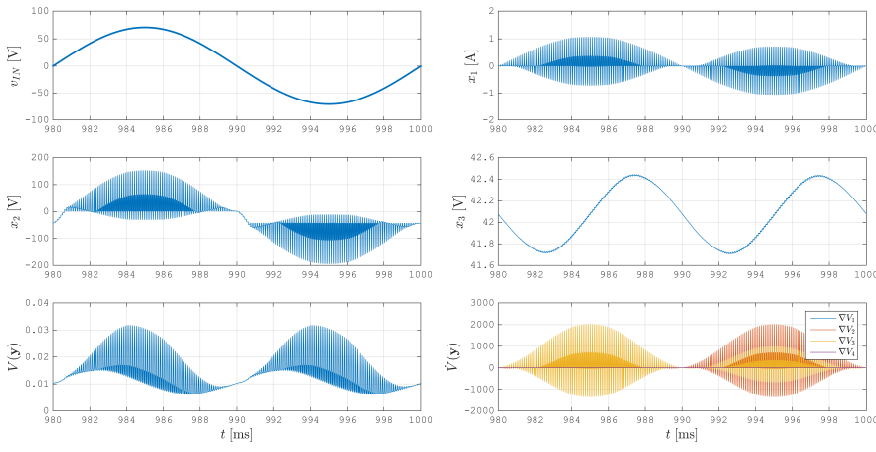

Fig. 5: Time diagrams during one switching period.

derivatives have the same value $\dot{V}_{2}(\mathbf{y})=\dot{V}_{3}(\mathbf{y})=\dot{V}_{4}(\mathbf{y})$. From subsystem 4 converter can only switch to combined subsystem $\widetilde{1}$ which is externally controlled. This completes the proof.

Theorem 1 states that for on and off positions of the controllable switch $\mathrm{S}$, the uncontrollable switches, being diodes D1 and D2, ensure the switching between subsystems such that the Lyapunov function derivative of the current subsystem is minimal, which is quite a remarkable characterization in technical terms. This also indicates through the Lyapunov analysis that the system stabilizes itself. Therefore, the analysis applies to the circuit's internal, that is, unknown unknown to a designer switchings. Furthermore, by computing the minimum Lyapunov function derivative in time, each operating subsystem can be characterized and thus accurately recognized. Therefore, we believe that this approach, based on the Lyapunov analysis, can be used for a successful hysteretic controller design [23].

Additionally, it should be noted that in the steady-state, the system may experience switching sequences with multiple occurrences of subsystems 2 and 3 , such as $\widetilde{1} \rightarrow 2 \rightarrow 3 \rightarrow 2 \rightarrow 4$ or $\widetilde{1} \rightarrow 3 \rightarrow 2 \rightarrow 3 \rightarrow 4$ etc., which happen when the condition for entering subsystem 4 is not satisfied. That means that one of the diodes D1 or D2 can conduct and the system enters subsystem 2 or 3, respectively, instead of subsystem 4 . This can be avoided by constructing AC-DC Ćuk converter with sufficiently large amplitude of the input voltage, as will be shown experimentally in the Section V.

\section{Simulation Results}

To demonstrate that the Lyapunov analysis fits the dynamical behavior of the converter, PFC rectifier's operation in the 
PLECS is simulated with the following circuit parameters: $L_{1}=1.1 \mathrm{mH}, C_{1}=68 \mathrm{nF}, C_{0}=390 \mu \mathrm{F}$ and $R=500 \Omega$. Losses on the diodes are given by $r_{d 1}=r_{d 2}=20 \mathrm{~m} \Omega$, $V_{D 1}=V_{D 2}=2 \mathrm{~V}$ and $r_{s}=50 \mathrm{~m} \Omega$. Input voltage is a sine wave with the line frequency $f_{0}=50 \mathrm{~Hz}$ and amplitude $V_{m}=60 \mathrm{~V}$. The simulation is performed with a constant frequency switch S PWM driver signal $f_{S}=10 \mathrm{kHz}$ and duty-ratio $d=0.2$. Obtained time diagrams are shown in Fig. 4 , which are zoomed to show one period $T_{0}$ in the steadystate in Fig. 5. Additionally, the quadratic Lyapunov function $V(\mathbf{y})$ with matrix $\mathbf{P}$ of the form (29) with $p=10$ was used and its values depicted, as well as its derivative values for all four subsystems, and denoted as $\nabla V_{i}$ for $i \in\{1,2,3,4\}$. The equilibrium was chosen as $\overline{\mathbf{x}}=\left[\begin{array}{lll}0 & V_{X} & V_{0}\end{array}\right]^{T}$, where $V_{X}=-21.8366 \mathrm{~V}$ and $V_{0}=43.6731 \mathrm{~V}$.

From Fig. 4, it can be seen that the Lyapunov function derivatives for subsystems 1 and 3 have the same sign which is opposite to subsystem 2 . Subsystem 4 has derivative values which are very small in the absolute value, thus approaching zero.

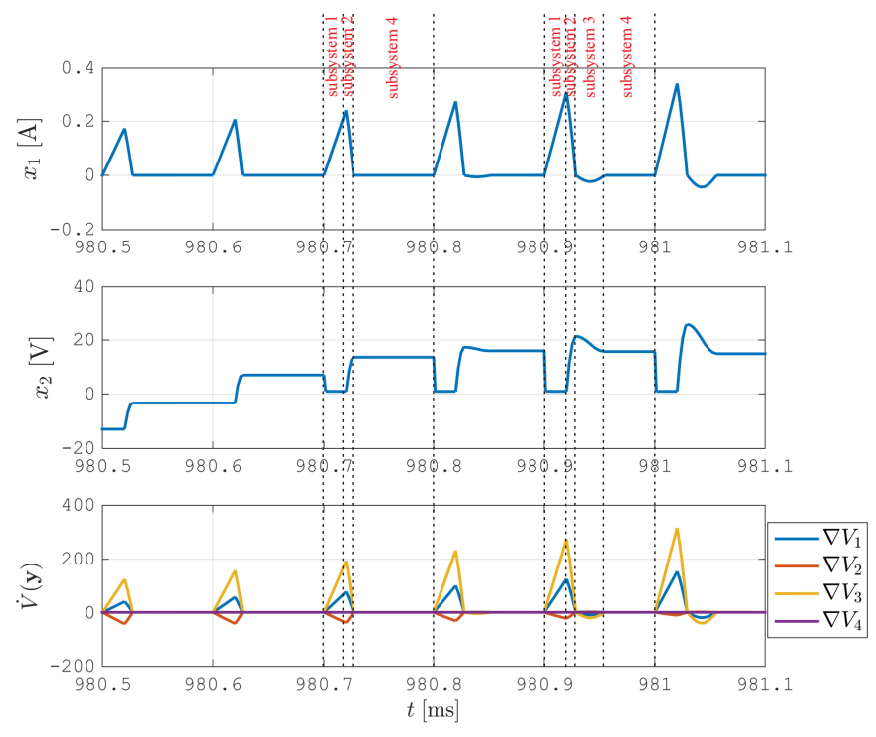

Fig. 6: Time diagrams for $v_{I N}>0$ and $t \in$ [980.5 ms, $981.1 \mathrm{~ms}]$.

Fig. 6 provides time diagrams of the state variables $x_{1}$ and $x_{2}$ and Lyapunov function derivatives' values denoted as $\nabla V_{i}$, $i=1,2,3,4$, when $v_{I N} \geq 0$ for $t \in[980.5 \mathrm{~ms}, 981.1 \mathrm{~ms}]$. In these diagrams, the converter switches first as $\widetilde{1} \rightarrow 2 \rightarrow 4$ and later $\widetilde{1} \rightarrow 2 \rightarrow 3 \rightarrow 4$.

\section{EXPERIMENTAL RESUlts}

The presented analysis is also tested using a laboratory prototype with the similar values of the circuit parameters as in the simulation, that is, the particular measured values of the circuit parameters are $L_{1}=1.1 \mathrm{mH}, C_{1}=68 \mathrm{nF}, C_{0}=390 \mu \mathrm{F}$ and $R=500 \Omega$. Furthermore, we used an input sinusoidal voltage source producing $v_{I N}=V_{m} \sin (100 \pi t)$. Diodes D1 and D2 are chosen as BYV29, while bidirectional switches are realized with transistors Q1 and Q2 being IRFP450, and diodes $\mathrm{D}_{Q 1}$ and $\mathrm{D}_{Q 2}$ are chosen as BY359. A difference between simulation and experiment was the value of inductor $L_{1}$. In the experimental setup this inductance was realized as discrete coil of approximately $100 \mu \mathrm{H}$ and inductance of autotransformer, which we used to lower the input voltage from 230 Vac. Also, line voltage in our city barely resembles sine wave. All this combined resulted in some differences between simulated and measured values yet given the outcomes indicate the same behaviors.

For the chosen input voltage with an amplitude of $V_{m}=$ $60 \mathrm{~V}$, the resulting output voltage is measured as $\bar{x}_{3}=37.1 \mathrm{~V}$, which is consistent with the simulation. From Fig. 7, it can be seen that the time diagrams during one period $T_{0}$ match the simulation results in Fig. 5. Fig. 8 shows results for the switching sequence $\widetilde{1} \rightarrow 2 \rightarrow 4$ around zero and then $\widetilde{1} \rightarrow 2 \rightarrow 3 \rightarrow 4$. In Fig. 9, time diagrams for the switching sequences resulting from $v_{I N} \gtrsim 0$, are shown. In Fig. 9a, the case when the sequence is $\tilde{1} \rightarrow 2 \rightarrow 3 \rightarrow 2 \rightarrow 4$ for $v_{I N}=60 \mathrm{~V} \sin (\omega t)$, is depicted, and in Fig. $9 \mathrm{~b}$, the case of sequence $\tilde{1} \rightarrow 2 \rightarrow 3 \rightarrow 4$ for $v_{I N}=70 \mathrm{~V} \sin (\omega t)$, is shown. These figures provide a clear validation that an increase of the input voltage causes the system not to enter subsystems 2 and 3 multiple times during one switching period.
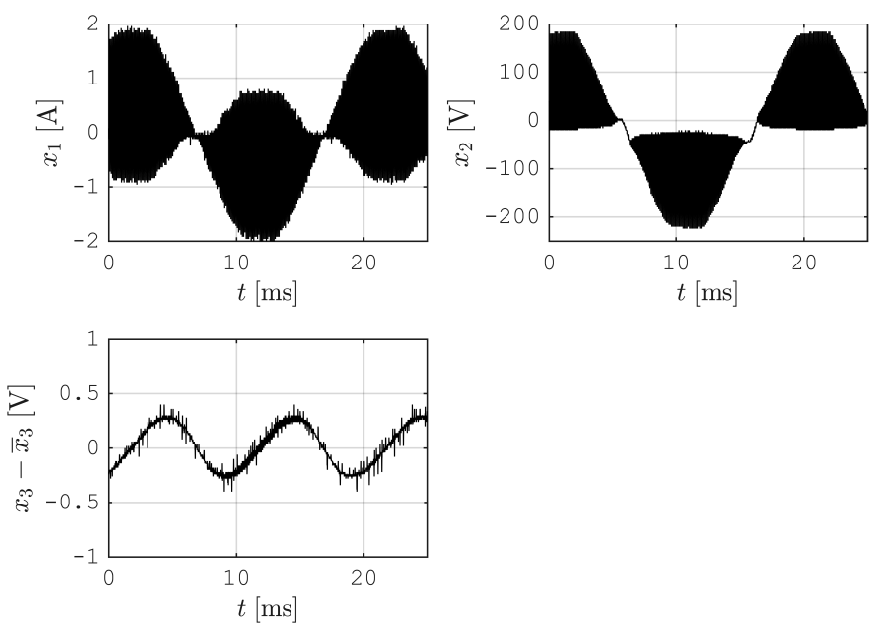

Fig. 7: Time diagrams of the state variables during one period of the input voltage measured by oscilloscope Tektronix TDS 1002B.

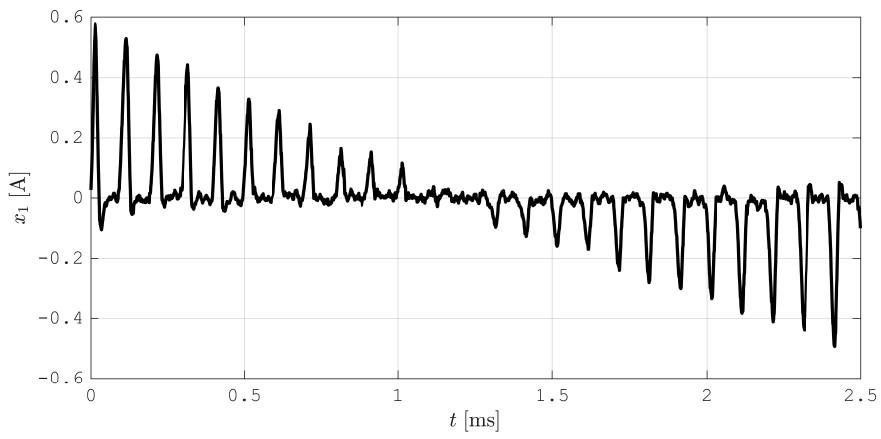

Fig. 8: Time diagram of the state variable $\mathrm{x} 1$ around zero crossing. 

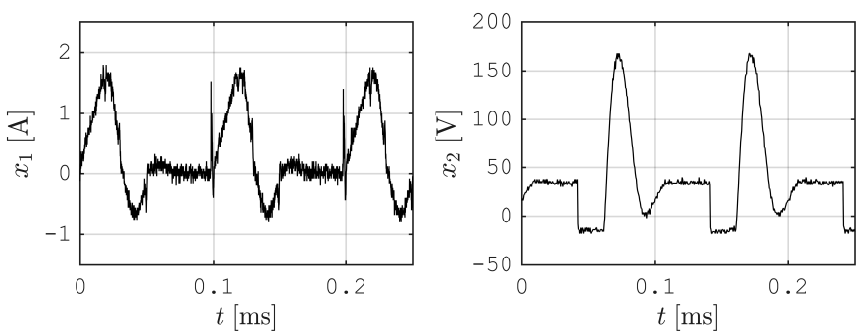

a)
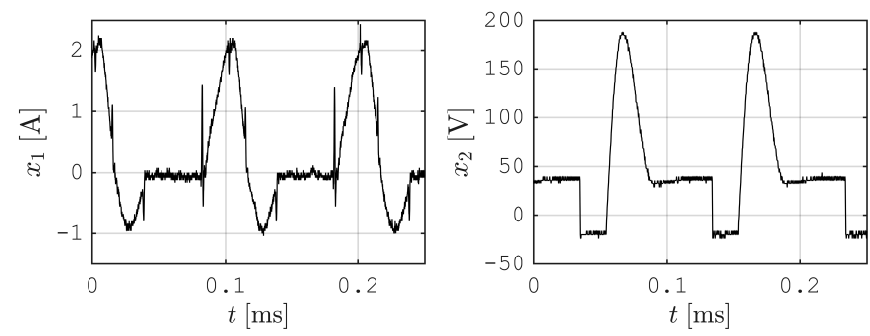

b)

Fig. 9: State variables $x_{1}$ and $x_{2}$ when the swithings are as follows: a) $\widetilde{1} \rightarrow 2 \rightarrow 3 \rightarrow 2 \rightarrow 4$ for $v_{I N}=60 \mathrm{~V} \sin (\omega t)$ and b) $\widetilde{1} \rightarrow 2 \rightarrow 3 \rightarrow 4$ for $v_{I N}=70 \mathrm{~V} \sin (\omega t)$.

\section{CONCLUSION}

In this work, a complete analysis and the characterization of the AC-DC Ćuk converter's operating modes and the switchings among them, have been provided. The switchings consist of the external switching dependent on the constant frequency and duty-ratio and the internal switchings which are linked to the minimum of a quadratic Lyapunov function's time derivatives related to different operating modes. The analysis is both illustrated and validated by a representative set of simulation and experimental results.

\section{ACKNOWLEDGEMENT}

Authors would like to thank prof. dr Milomir Šoja, prof. dr Slobodan Lubura and Srđan Lale from the Faculty of Electrical Engineering, University of East Sarajevo, for their valuable suggestions in building the experimental setup.

This work was partially supported by the project TR33020 of the Ministry of Education, Science and Technological Development of the Republic of Serbia.

\section{REFERENCES}

[1] M. N. Z. Abidin, "IEC 61000-3-2 harmonics standards overview," Schaffner EMC Inc., Edsion, NJ, USA, 2006.

[2] K. Sakthivel, S. K. Das, and K. Kini, "Importance of quality AC power distribution and understanding of emc standards IEC 61000-3-2, IEC 61000-3-3 and IEC 61000-3-11," in Electromagnetic Interference and Compatibility, 2003. INCEMIC 2003. 8th International Conference on. IEEE, 2003, pp. 423-430.

[3] I. E. Commission et al., "Electromagnetic compatibility (EMC)-part 34: Limits-limitation of emission of harmonic currents in low-voltage power supply systems for equipment with rated current greater than 16 A," IEC, vol. 61000, pp. 3-4, 1998.

[4] S. Ćuk, "Bridgeless PFC converter," Oct. 14 2010, uS Patent App. 12/798,682. [Online]. Available: https://www.google.com/patents/US20100259240
[5] —, "Single-stage AC-to-DC converter with isolation and power factor correction," Dec. 1 2011, uS Patent App. 12/802,122. [Online]. Available: https://www.google.com/patents/US20110292703

[6] —_, "Bridgeless PFC converter achieves $98 \%$ efficiency, 0.999 power factor," Power, vol. 4, no. 6.0, pp. 8-0, 2010.

[7] - "Single-stage bridgeless isolated PFC converter achieves $98 \%$ efficiency," Power Electronics Technology, pp. 22-31, 2010.

[8] M. Mahdavi and H. Farzanehfard, "Bridgeless SEPIC PFC rectifier with reduced components and conduction losses," IEEE Transactions on Industrial Electronics, vol. 58, no. 9, pp. 4153-4160, 2011.

[9] E. H. Ismail, "Bridgeless SEPIC rectifier with unity power factor and reduced conduction losses," IEEE Transactions on Industrial Electronics, vol. 56, no. 4, pp. 1147-1157, 2009.

[10] A. M. Al Gabri, A. A. Fardoun, and E. H. Ismail, "Bridgeless PFCmodified SEPIC rectifier with extended gain for universal input voltage applications," IEEE Transactions on Power Electronics, vol. 30, no. 8, pp. 4272-4282, 2015.

[11] V. Bist and B. Singh, "An adjustable-speed PFC bridgeless Buck-Boost converter-fed BLDC motor drive," IEEE Transactions on Industrial Electronics, vol. 61, no. 6, pp. 2665-2677, 2014.

[12] H. Wang, Y. Tang, and A. Khaligh, "A bridgeless Boost rectifier for lowvoltage energy harvesting applications," IEEE transactions on power electronics, vol. 28, no. 11, pp. 5206-5214, 2013.

[13] W.-Y. Choi, J.-M. Kwon, E.-H. Kim, J.-J. Lee, and B.-H. Kwon, "Bridgeless Boost rectifier with low conduction losses and reduced diode reverse-recovery problems," IEEE Transactions on Industrial Electronics, vol. 54, no. 2, pp. 769-780, 2007.

[14] L. Huber, Y. Jang, and M. M. Jovanovic, "Performance evaluation of bridgeless PFC boost rectifiers," IEEE Transactions on Power Electronics, vol. 23, no. 3, pp. 1381-1390, 2008.

[15] D. S. L. Simonetti, J. Viera, and G. C. D. Sousa, "Modeling of the high-power-factor discontinuous Boost rectifiers," IEEE transactions on industrial electronics, vol. 46, no. 4, pp. 788-795, 1999.

[16] U. Anwar, R. Erickson, D. Maksimović, and K. K. Afridi, "A control architecture for low current distortion in bridgeless Boost power factor correction rectifiers," in Applied Power Electronics Conference and Exposition (APEC), 2017 IEEE. IEEE, 2017, pp. 82-87.

[17] M. Bodetto, A. El Aroudi, A. Cid-Pastor, and M. Al-Numay, "Improving the dimming performance of low-power single-stage AC-DC HBLED drivers," IEEE Transactions on Industrial Electronics, 2017.

[18] K. Yao, X. Ruan, X. Mao, and Z. Ye, "Reducing storage capacitor of a DCM Boost PFC converter," IEEE Transactions on Power Electronics, vol. 27, no. 1, pp. 151-160, 2012.

[19] D. S. L. Simonetti, J. Sebastian, and J. Uceda, "The discontinuous conduction mode Sepic and Cuk power factor preregulators: analysis and design," IEEE Transactions on Industrial Electronics, vol. 44, no. 5, pp. 630-637, 1997.

[20] A. A. Fardoun, E. H. Ismail, M. A. Al-Saffar, and A. J. Sabzali, "A bridgeless resonant pseudoboost PFC rectifier," IEEE Transactions on Power Electronics, vol. 29, no. 11, pp. 5949-5960, 2014.

[21] J. C. Mayo-Maldonado and P. Rapisarda, "Dissipative switched linear differential systems," IEEE Transactions on Automatic Control, vol. 61, no. 12, pp. 3813-3825, 2016.

[22] A. A. Fardoun, E. H. Ismail, M. A. Al-Saffar, and A. J. Sabzali, "New âĂIJrealâĂİ bridgeless high efficiency AC-DC converter," in Applied Power Electronics Conference and Exposition (APEC), 2012 TwentySeventh Annual IEEE. IEEE, 2012, pp. 317-323.

[23] A. Lekić and D. Stipanović, "Hysteresis switching control of the Ćuk converter," IEEE Transactions on Circuits and Systems I: Regular Papers, vol. 63, no. 11, 2016.

[24] A. Lekić and D. Stipanović, "Hysteresis switching control of the Ćuk converter operating in discontinuous conduction modes," IEEE Transactions on Circuits and Systems II: Express Briefs, vol. 64, no. 9, 2016.

[25] A. F. Filippov, Differential Equations with Discontinuous Righthand Sides. Dordrecht, The Netherlands: Kluwer Academic Publishers, 1988. 


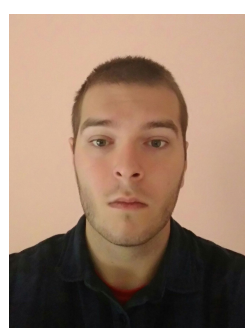

Nikola Ž. Petrović received Bachelor (2016) and M.Sc. degree (2017) in Electrical and Computer Engineering from the School of Electrical Engineering, University of Belgrade, Serbia. He is currently a Teaching and Research Assistant at the same school, where he is also a Ph.D. student in the Department of Electronics.

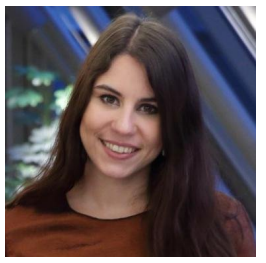

Aleksandra Lekić received B.S., M.S. and Ph.D degrees in Electrical Engineering from the University of Belgrade, in 2012, 2013 and 2017, respectively. Since December 2012, she works as teaching assistant and since 2018 as an assistant professor at the School of Electrical Engineering, University of Belgrade.

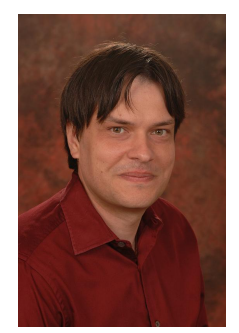

Dušan M. Stipanović received his undergraduate degree in electrical engineering from the University of Belgrade in 1994, and graduate degrees (advised by Dr. Dragoslav Šiljak) in electrical engineering from Santa Clara University in 1996 and 2000, respectively. He had been a Research Associate in the Department of Aeronautics and Astronautics at Stanford University between 2001 and 2004 (supervised by Dr. Claire Tomlin). He is an Associate Professor in the ISE Department and Coordinated Science Laboratory at the University of Illinois at Urbana-Champaign and a visiting Professor in the School of Electrical Engineering, University of Belgrade, Serbia. Dr. Stipanović served as an Associate Editor on the Editorial Boards of the IEEE Transactions on Circuits and Systems I and II. Currently he is an Associate Editor for Journal of Optimization Theory and Applications. 